\title{
Segmentación Portal Hepática Mediante Imágenes Tomográficas Tridimensionales Reconstituidas
}

\author{
Hepatic Portal Segmentation Using Reconstructed Three-Dimensional Tomography Images
}

César Augusto Durand López ${ }^{1}$; Carlos Alberto Torres Girón² \& Ademar Cervera Carrillo

DURAND, L. C. A.; TORRES, G. C.A. \& CERVERA, C. A. Segmentación portal hepática mediante imágenes tomográficas tridimensionales reconstituidas. Int. J. Morphol., 35(3):859-863, 2017.

RESUMEN: La descripción de las imágenes tridimensionales reconstituidas de tomografías hepáticas (Three-dimensional computed tomographic volume rendering images) tiene alto porcentaje de variaciones anatómicas inexplicables, de tal manera que no orientan en forma definitiva al radiólogo y al cirujano. Este estudio permite ver y describir las ramas segmentarias de la vena porta hepática (A12.3.12.001)*, su relación con las venas hepáticas (A12.3.09.005) y su distribución parenquimal. El estudio es descriptivo y usamos expresiones porcentuales para citar la frecuencia de las características anatómicas, para esto tomamos al azar 56 reconstrucciones tridimensionales de tomografías hepáticas de pacientes adultos. Las ramas segmentarias portales+ y las venas hepáticas fueron identificadas siempre; hallamos siete ramas segmentarias portales en todos los casos, además, que la rama portal segmentaria $\mathrm{V}$ (cinco)+ nació de la porta derecha (A12.3.12.002) en 75 $\%$ de casos y de la izquierda (A12.3.12.005) en 21,4\%.

PALABRAS CLAVE: Imágenes tomográficas reconstituidas; Hígado; Segmentación portal.

\section{INTRODUCCIÓN}

Los estudios radiológicos modernos (tomografía, resonancia magnética) ofrecen buenas imágenes del cuerpo humano. Sin embargo, respecto al hígado (A05.8.01.001), los conceptos anatómicos actuales no logran explicar la anatomía de este órgano, así tenemos que las definiciones, esquemas y conocimientos en los textos de medicina en relación al hígado confunden al estudiante, al médico y especialmente al radiólogo y al cirujano, los cuales, en su afán por superar esta "Babel" de términos hepáticos, acordaron una terminología basada en lo publicado y no en estudios anatómicos: "Terminology Committee of the International HepatoPancreato-Biliary Association: The Brisbane 2000 Terminology of Liver Anatomy and Resections" (Belgihiti et al., 2000), sin embargo, ninguno de estos acuerdos logra disminuir la creciente cantidad de trabajos que manifiestan la falta de conocimientos anatómicos acerca del hígado. La solución es sencilla, aquí ofrecemos un aporte.

\section{MATERIAL Y MÉTODO}

Se evaluaron 56 estudios tomográficos de hígado de pacientes adultos de ambos sexos, todas las razas. Las reconstrucciones tridimensionales y las tomografías fueron hechas con un tomógrafo espiral multicorte Phillips modelo Brilliance CT64. La técnica tomográfica fue en tres fases (arterial tardía, venosa portal, y fase tardía) obtenidas 25 segundos, 45 segundos, y 75 segundos después de iniciada la inyección del contraste; usamos un multidetector helicoidal para la tomografía computarizada. Los cortes fueron de $0,625 \mathrm{~mm}$ de grosor. El contraste usado fue Iopamidol $370 \mathrm{mg} / \mathrm{mL}$. La dosis usada fue $1,5 \mathrm{~mL}(555 \mathrm{mg}) / \mathrm{k}$ de peso.

Mediante barrido y limpieza del parénquima hepático en las imágenes reconstituidas pudimos ver la vena porta hepática, sus ramas primarias izquierda y derecha, las ramas secundarias segmentarias terminales+ que emergen de cada rama primaria y las venas hepáticas.

Cuando un término es mencionado por primera vez en el texto, usamos el número de identificación de Terminologia Anatomica Internacional. Cerramos entre paréntesis los elementos inconstantes y marcamos con + aquellos términos no cubiertos por Terminologia Anatomica Internacional.

\footnotetext{
${ }^{1}$ Cirujano del Hospital de Emergencias "José Casimiro Ulloa”, Profesor de Anatomía Humana, Facultad de Medicina, Universidad Ricardo Palma, Lima,Perú.

${ }^{2}$ Tecnólogo Médico especialista en Tomografías, Hospital "José Casimiro Ulloa”, Lima, Perú.

${ }^{3}$ Tecnólogo Médico especialista en muestras anatómicas, Universidad Ricardo Palma, Lima, Perú.
} 


\section{RESULTADOS}

Observamos siete ramas portales segmentarias en todos los casos, de la siguiente manera:

Rama segmentaria I y subsegmentarias: en 48/52 (75 $\%$ ) nacieron de ambas ramas portales, izquierda y derecha, 2/ $52(3,8 \%)$ fueron solamente izquierdas y 2/52 (3,8\%) nacieron de la rama izquierda y directamente de la vena porta. En 4/56 fue difícil identificar esta rama segmentaria.

Ramas segmentarias II, III y IV fueron izquierdas en todos los casos: 56/56 (100\%).

La rama segmentaria $\mathrm{V}$ fue derecha (Fig. 1) en 42/56 $(75 \%)$ e izquierda (Fig. 2) en 12/56 (21,4\%), en 2/56 (3,57 $\%)$ nació directamente de la vena porta.

Las ramas segmentarias VI y VII fueron derechas siempre, dentro de estas, en 2/56 (3,57\%) casos la rama VI nació de la $\mathrm{V}$ y esta de la porta derecha.

El segmento I (A05.8.01.044) (lóbulo caudado) recibió una rama de la porta izquierda en $100 \%$ de casos, además, en $75 \%$ recibió una rama de la porta derecha, y en 3,8 \% recibió una rama de la vena porta hepática. Estas ramas nacieron en el margen posterior de las ramas portales primarias, en ángulo recto y corrieron hacia atrás horizontalmente.

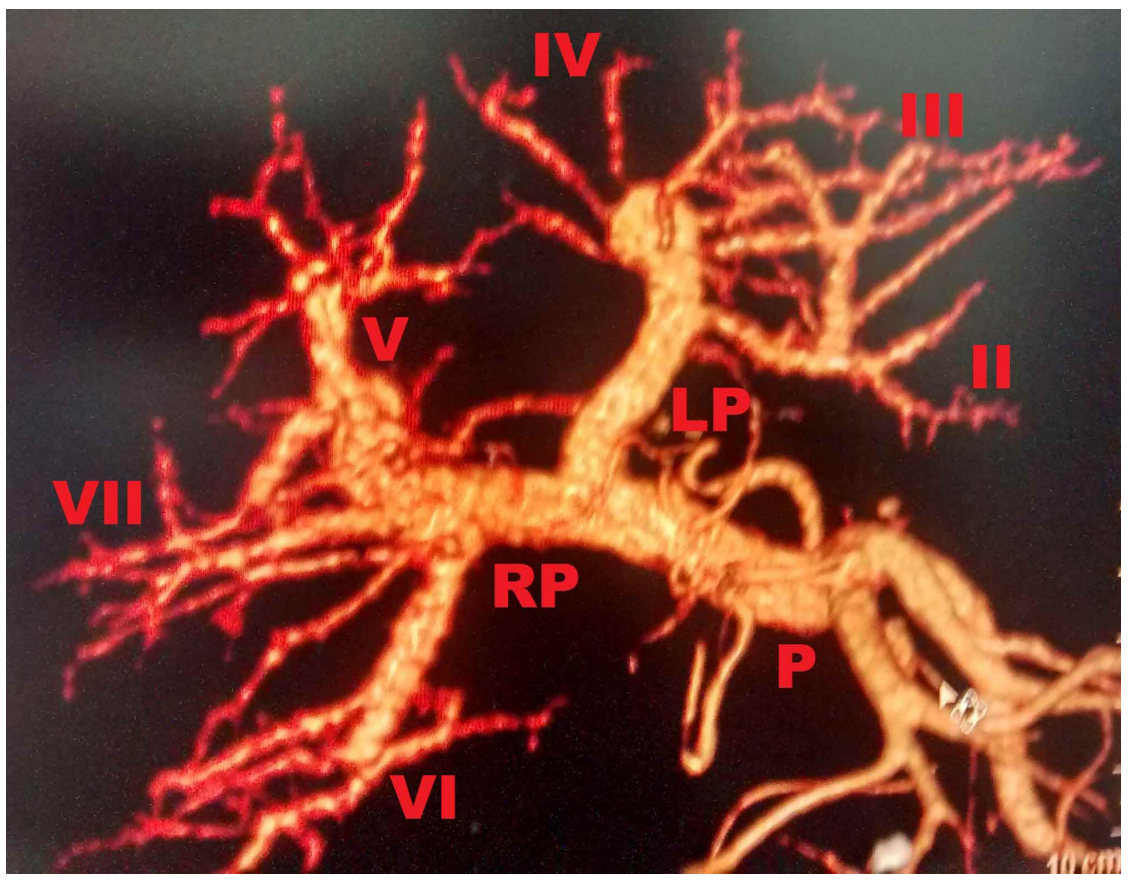

Fig. 1. Imagen reconstituida, vena Porta (P) y su rama derecha (RP) e izquierda (LP), la rama segmentaria $\mathrm{V}$ nació de la rama derecha.

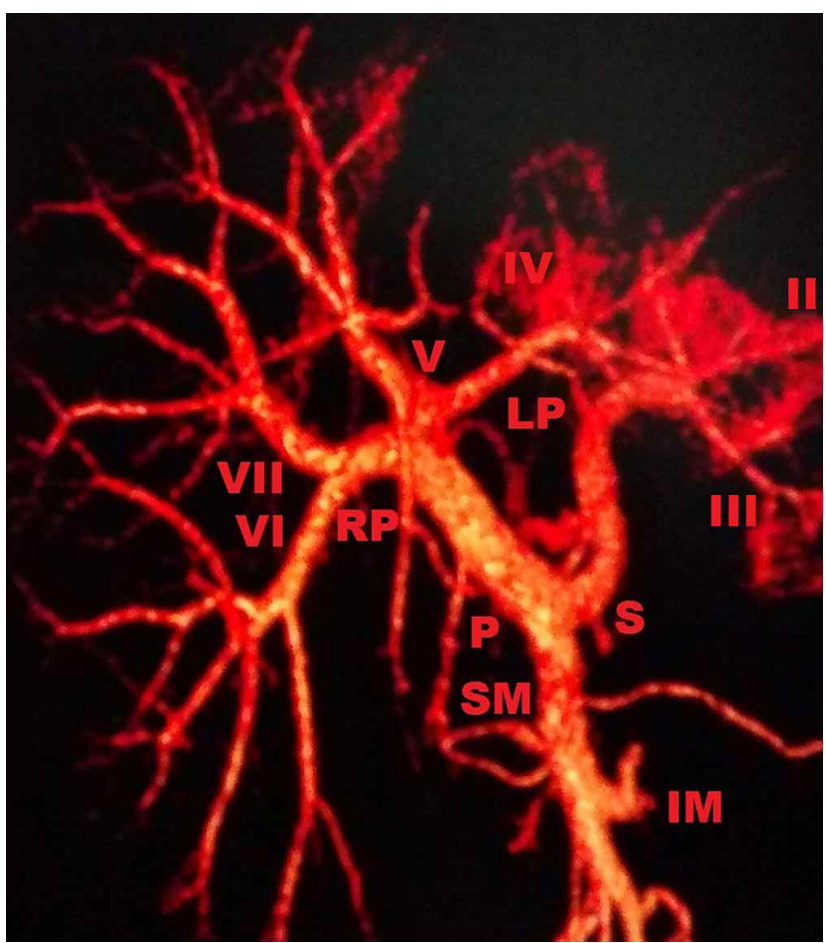

Fig. 2. Imagen reconstituida, vena Porta $(\mathrm{P})$ y su rama derecha (RP) e izquierda (LP), la rama segmentaria V nació de la rama izquierda.
La rama para el segmento II (A05.8.01.039) nació en el ángulo que forman la porción transversal (A12.3.12.006) y la umbilical (A12.3.12.008) de la porta izquierda. La rama II formó con la porción transversa de la porta izquierda un ángulo de más o menos 135 grados abierto hacia atrás, esta rama apuntó hacia atrás y afuera (izquierda).

La rama para el segmento III (A05.8.01.040) emergió de la izquierda del bulbo terminal de la porción umbilical de la porta izquierda, apuntó hacia adelante y afuera (izquierda), siempre se acompañó de ramas subsegmentarias+ de menor calibre.

La rama para el segmento IV (A05.8.01.042) nació a la derecha del extremo bulbar de la porción umbilical de la porta izquierda, se acompañó de un ramillete de ramas subsegmentarias [que abarcaron el parénquima entre la fisura 
umbilical (A05.8.01.034) y la fisura portal principal (A05.8.01.035), desde el margen hepático inferior (A05.8.01.024) hasta la vena cava inferior (A12.3.09.001), volumen en forma de cuña, angosto por la cara inferior y que se va abriendo hacia la cara diafragmática (A05.8.01.002) con una divergencia de 20 grados entre sus paredes. La cara inferior del segmento tiene tres porciones: la anterior que corresponde al lóbulo cuadrado (A05.8.01.029) y mitad izquierda de la fosa de la vesícula biliar

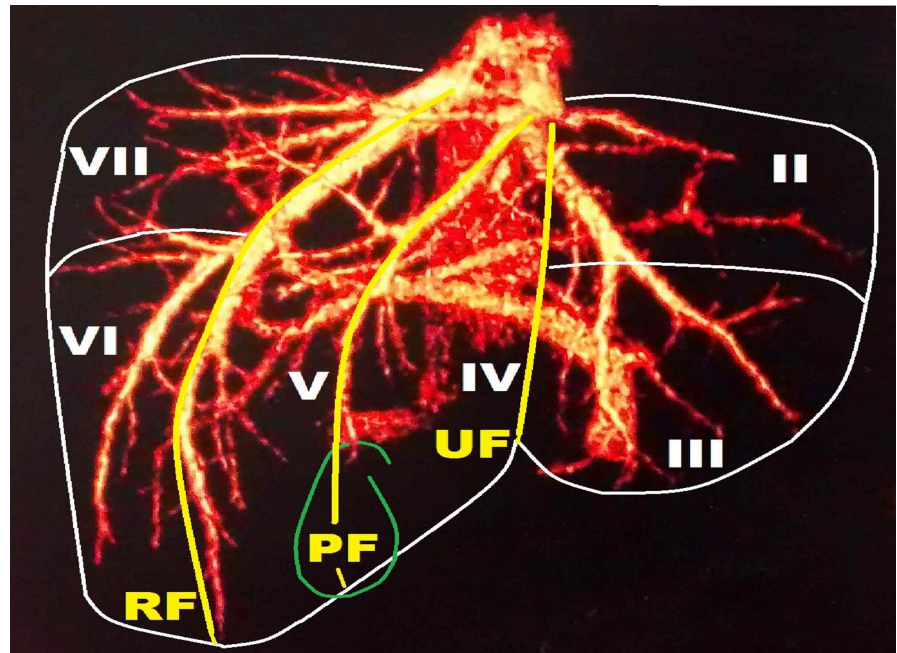

Fig. 3. Imagen reconstituida, venas hepáticas, la fisura derecha (RF) está ocupada por la vena hepática derecha, la fisura principal (PF) por la vena intermedia, la fisura umbilical (UF) no contiene vena de retorno, la vena hepática izquierda nace en el centro del segmento III y atraviesa el segmento II antes de desembocar en la vena cava inferior.
(A05.8.01.013), la media que pasa sobre la rama izquierda de la porta; y la porción posterior que se apoya sobre la mitad izquierda de la cara superior del lóbulo caudado]. En algunos casos, las ramas portales subsegmentarias que irrigan la porción suprayacente al lóbulo caudado nacieron en el margen superior de la porción transversa de la porta izquierda.

La rama para el segmento $\mathrm{V}$ (A05.8.01.047) nació en el margen superior de la porta derecha, a $1 \mathrm{~cm}$ de la bifurcación portal, o en el margen superior de la porta izquierda, a $0.5 \mathrm{~cm}$ de la bifurcación portal. En ambos casos se dirigió hacia la cúpula hepática dando ramas anteriores, laterales, posteriores y mediales (abarcó el territorio comprendido entre la fisura derecha y la principal, desde el margen inferior del hígado hasta la cava inferior por detrás, este segmento tuvo forma de cono invertido, sus paredes divergen noventa grados).

La rama para el segmento VI (A05.8.01.050) nació en el extremo lateral de la porta derecha y se dirigió directo hacia el ángulo hepático derecho, con una trayectoria de arriba hacia abajo, de atrás hacia adelante y de adentro hacia afuera.

La rama para el segmento VII (A05.8.01.051) nació del extremo lateral de la porta derecha, describió una curva de concavidad medial, hacia atrás, arriba y adentro, como si tratara de abrazar a la vena cava inferior.

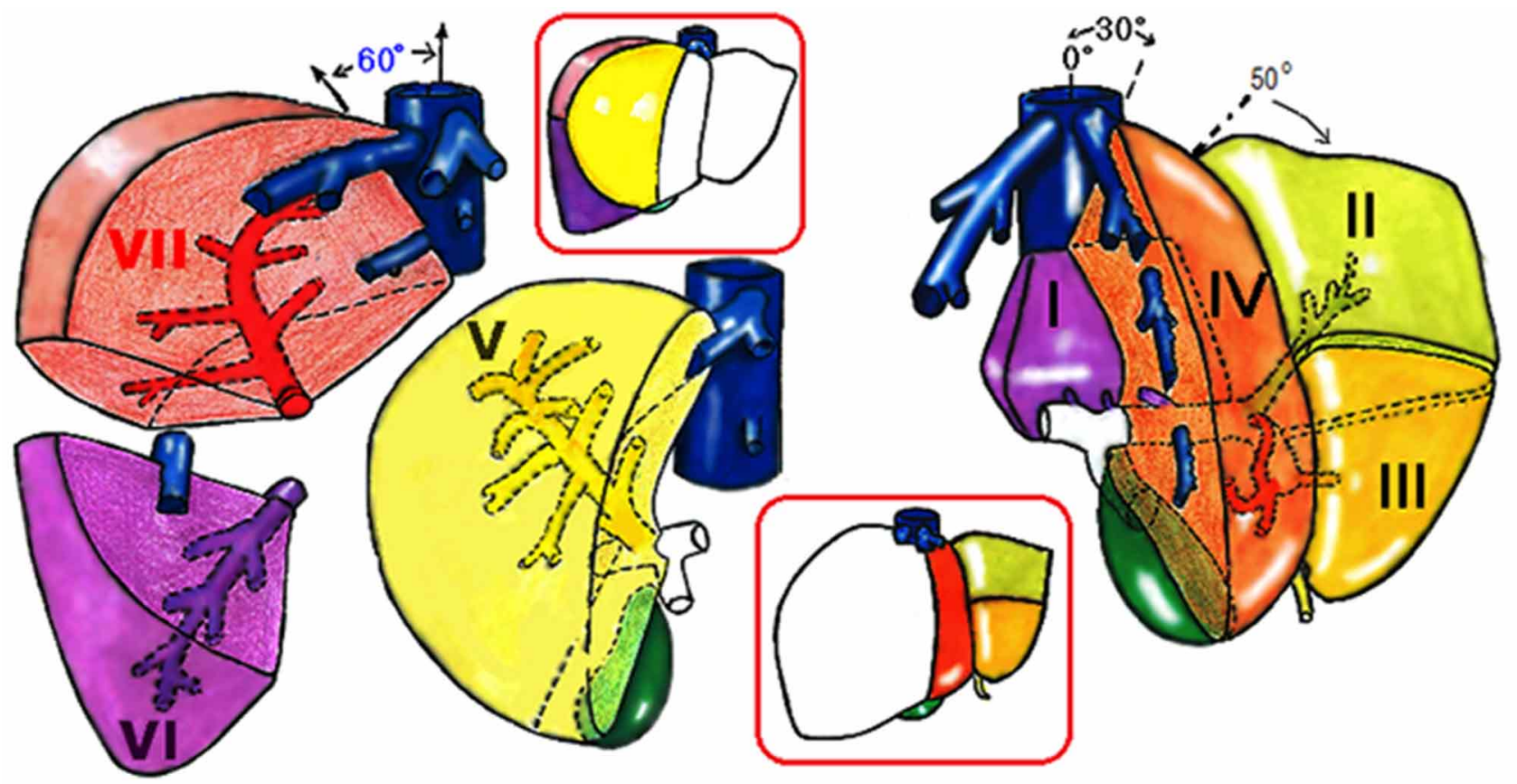

Fig. 4. Esquema de los segmentos del lóbulo derecho e izquierdo, planos de inclinación de las fisuras portales y su contenido. 
Identificamos también (Fig. 3) la vena hepática derecha (ocupa la fisura portal derecha), la vena hepática intermedia (ocupa la fisura portal principal) y la vena hepática izquierda (no ocupa la fisura umbilical, sino transcurre dentro de los segmentos III y II).

De esta manera, las siete ramas segmentarias van a sus respectivos territorios parenquimales, los cuales están limitados por las fisuras portales verticales y horizontales+, se forman así cuatro divisiones (A05.8.01.033) limitadas por las tres fisuras verticales conteniendo los segmentos en el siguiente orden:

Lóbulos izquierdo (A 05.8.01.027) y derecho (A05.8.01.026) separados por la fisura portal principal. El izquierdo es subdividido verticalmente en dos por el plano de la fisura umbilical; el derecho es subdividido verticalmente en dos por el plano de la fisura derecha.

Lóbulo izquierdo: División lateral (A05.8.01.038), con los segmentos II y III a la izquierda de la fisura umbilical, la vena hepática izquierda atraviesa el segmento III y el II. La División medial (A05.8.01.041), con el segmento IV medial a la fisura umbilical.

Lóbulo derecho: División lateral (A05.8.01.049), con los segmentos VI y VII laterales a la fisura derecha. La División medial (A05.8.01.046), con el segmento V medial a la fisura derecha. La cara inferior de la mitad posterior de los segmentos IV y V descansa sobre el segmento I (Cho et al., 2000). Los segmentos IV y V tienen en común que se ubican a izquierda y derecha de la fisura portal principal respectivamente, se extienden desde el margen hepático inferior hasta la vena cava inferior por detrás (Fig. 4).

\section{DISCUSIÓN}

Desde el siglo pasado hasta hoy, autores como Sexton \& Zeman (1983), Soyer et al. (1995), Rutkauskas et al. (2006), Altun et al. (2015) compararon la anatomía hepática con las imágenes tomográficas usando como marcadores anatómicos a las venas hepáticas (Scheuerlein \& Köckerling, 2000) y al ligamento redondo. Soyer et al. (1995) en 60 de 69 casos (87 $\%)$ observaron las venas hepáticas derecha, intermedia e izquierda, en 19 casos (32\%) venas hepáticas accesorias y en $6(9 \%)$ venas hepáticas inferiores. Se encontraron variaciones en la anatomía portal intrahepática en 4 pacientes $(6 \%)$ que consistían en la trifurcación de la vena porta en tres pacientes (4 \% ). van Leeuwen et al. (1995) señalaron "In the simplified Couinaud classification, in which the liver is divided into eight segments, each supplied by a central vasculo-biliary sheath, little attention is given to the high prevalence of anatomical variations which occur, especially in the right hemiliver"; nuestro trabajo mostró que el lóbulo derecho está formado por los segmentos V, VI y VII en $75 \%$ de casos y por los segmentos VI y VII en 21,4 \%, en estos últimos la rama portal del segmento V nació de la porta izquierda. Fasel et al. (1998) en su trabajo "Segmental anatomy of the liver. Poor correlation with CT" encontraron en 100 hígados estudiados mediante tomografía computarizada helicoidal y comparados con los auténticos territorios anatómicos de distribución un porcentaje de error de 51,6\% +/- 19.9. Botero \& Strasberg (1998) en su trabajo "Division of the left hemiliver in man, segments, sectors, or sections" concluyeron que sus resultados diferían del sistema "sectorial" propuesto por Couinaud en el lado izquierdo del hígado. La división del lóbulo izquierdo por la fisura umbilical que fue propuesta por Healey es anatómicamente lógica y describe adecuadamente las resecciones quirúrgicas comunes. Hata et al. (1999) encontraron en 197 hígados de adultos disecados por su cara inferior, que los segmentos VI y VII del lóbulo derecho coincidieron con la descripción hecha por Couinaud; también sugirieron que las venas hepáticas (derechas, intermedia e izquierda) ayudan a identificar las ramas portales segmentarias. Manifestaron dificultad en identificar el margen inferior del sector lateral derecho y la porción postero-inferior del hígado en 30$40 \%$ de casos. Estos tres últimos autores encontraron dificultad en describir la anatomía tomográfica del hígado.

Baba et al. (2000) y Wu et al. (2007) entre otros, describieron casos de tripartición de la porta al ingresar al hígado; en estos casos la rama portal $\mathrm{V}$ debió nacer de la porta izquierda, si hubieran buscado la rama $\mathrm{V}$ en el lado derecho no la hubieran encontrado, esta impresión se debió al nacimiento de la rama $\mathrm{V}$ al inicio de la porta izquierda, a $0.5 \mathrm{~cm}$ de la bifurcación portal, con un diámetro y una longitud parecidos a los de la porta izquierda (Fig. 2).

Mildenberger et al. ( 2000) señalaron lo inseguro que es usar la división de Couinaud para los estudios tomográficos de hígado. Ishibashi et al. (2001) manifestaron que la clasificación de Couinaud no coincide con la segmentación del hemihígado izquierdo. Fischer et al. (2002) manifestaron que la clasificación de Couinaud es buena para una descripción anatómica "grosso modo", pero no explica muchas variaciones. Rieker et al. (2003) señalaron que en 51 de 409 casos $(12,5 \%)$ de tomografías hepáticas reconstituidas no fueron identificados plenamente usando la clasificación de Couinaud. Castorina (2014) en su "Review of the nomenclature of the liver anatomical and functional areas by three dimensional volume rendering 64-multislice computed tomography. Proposal for an update of the terminology" dijeron: "In the last centuries, the anatomy of the liver has been the object of increasing interest. The International Anatomical Terminology 
tries to unify the terminology of liver anatomy, making it a living language. A single, worldwide-accepted classification of the liver still does not exist. In fact, definition of segments according to Couinaud's nomenclature is different from that of Goldsmith and Woodburne". Las imágenes reconstituidas de nuestros 56 casos mostraron 7 ramas portales segmentarias siempre, en 12 casos la rama portal $\mathrm{V}$ nació de la porta izquierda, en 42 de la porta derecha y en 2 casos continuó el eje de la vena porta. La descripción de las imágenes concuerda $100 \%$ con la anatomía mostrada en los acrílicos de hígado en estudios anteriores (Durand, 2016, 2017), estas correlaciones nos permiten inferir que el estudio de preparados acrílicos de hígado es muy útil para interpretar las imágenes tomográficas tridimensionales reconstituidas (Fig. 4).

En conclusión, las imágenes tomográficas tridimensionales reconstituidas del hígado permiten reconocer siete ramas portales segmentarias y las venas hepáticas (con la única variación en el nacimiento de la rama portal $\mathrm{V}$, que puede ser derecha o izquierda), con esta información podemos ubicar los territorios segmentarios.

DURAND, L. C.A.; TORRES, G. C. A. \& CERVERA, C. A. Hepatic portal segmentation using reconstructed three-dimensional tomography images. Int. J. Morphol., 35(3):859-863, 2017.

SUMMARY: The anatomical description of reconstructed three-dimensional images of hepatic CT scans (Three-dimensional computed tomographic volume rendering images) has a high percentage of unexplained anatomical variations, so that they do not definitively orient the radiologist and the surgeon. This study allows to observe and to describe the segmental branches of the Hepatic portal vein (A12.3.12.001), its relation with the hepatic veins (A12.3.09.005) and its parenchymal distribution. The study is descriptive and we used percentage expressions to describe the frequency of anatomical characteristics. For this we randomly took 56 three-dimensional reconstructions of liver CTs from adult patients. Portal segmental branches+ and hepatic veins were always identified; we found seven portal segmental branches in all cases. In addition, the segmental portal branch $\mathrm{V}$ (five)+ originated from the right portal (A12.3.12.002) in $75 \%$ of cases and from the left (A12.3.12.005) in $21.4 \%$.

KEY WORDS: Reconstructed tomographic images; Liver; Portal segmentation.

\section{REFERENCES}

Altun, E.; El-Azzazi, M. \& Semelka, R. C. (Eds.). Liver Imaging: MRI with CT Correlation. Oxford, Wiley-Blackwell, 2015.

Baba, Y.; Hokotate, H.; Nishi, H.; Inoue, H. \& Nakajo, M. Intrahepatic portal venous variations: demonstration by helical CT during arterial portography. J. Comput. Assist. Tomogr., 24(5):802-8, 2000.

Belgihiti, J.; Clavien, P. A.; Gadzijev, E.; Garden, J. O.; Lau, W. Y. \& Makuuchi, M. The Brisbane 2000 Terminology of Liver Anatomy and Resections Terminology Committee of the International Hepato-Pancreato-Biliary Association: Chairman, SM Strasberg (USA). HPB, 2(3):333-9, 2000.
Botero, A. C. \& Strasberg, S. M. Division of the left hemiliver in man-segments, sectors, or sections. Liver Transpl. Surg., 4(3):226-31, 1998.

Castorina, S. Review of the nomenclature of the liver anatomical and functional areas by three-dimensional volume rendering 64-multislice computed tomography. Proposal for an update of the terminology. Ital. J. Anat. Embryol., 119(3):169-79, 2014.

Cho, A.; Okazumi, S.; Takayama, W.; Takeda, A.; Iwasaki, K.; Sasagawa, S.; Natsume, T.; Kono, T.; Kondo, S.; Ochiai, T. \& Ryu, M. Anatomy of the right anterosuperior area (segment 8 ) of the liver: evaluation with helical CT during arterial portography. Radiology, 214(2):491-5, 2000.

Durand, L. C. A. Liver: Seven hepatic segments. Int. J. Morphol., 34(4):152230, 2016.

Durand, L. C. A. Liver: The portal segment V. Int. J. Morphol., 35(1):363-7, 2017.

Fasel, J. H.; Selle, D.; Evertsz, C. J.; Terrier, F.; Peitgen, H. O. \& Gailloud, P. Segmental anatomy of the liver: poor correlation with CT. Radiology, 206(1):151-6, 1998.

Fischer, L.; Cardenas, C.; Thorn, M.; Benner, A.; Grenacher, L.; Vetter, M.; Lehnert, T.; Klar, E.; Meinzer, H. P. \& Lamadé, W. Limits of Couinaud's liver segment classification: a quantitative computer-based three-dimensional analysis. J. Comput. Assist. Tomogr., 26(6):962-7, 2002.

Hata, F.; Hirata, K.; Murakami, G. \& Mukaiya, M. Identification of segments VI and VII of the liver based on the ramification patterns of the intrahepatic portal and hepatic veins. Clin. Anat., 12(4):229-44, 1999.

Ishibashi, Y.; Sato, T. J.; Hirai, I.; Murakami, G.; Hata, F. \& Hirata, K. Ramification pattern and topographical relationship between the portal and hepatic veins in the left anatomical lobe of the human liver. Okajimas Folia Anat. Jpn., 78(2-3):75-82, 2001.

Rieker, O.; Klos, G.; Beckmann, P.; Vomweg, T. W.; Otto, G. \& Thelen, M. Automatic classification of liver segments according to Couinaud: development of a new algorithm and evaluation spiral CT data. Rofo, 175(12):1655-9, 2003.

Rutkauskas, S.; Gedrimas, V.; Pundzius, J.; Barauskas, G. \& Basevicius, A. Clinical and anatomical basis for the classification of the structural parts of liver. Medicina (Kaunas), 42(2):98-106, 2006.

Scheuerlein, H. \& Köckerling, F. Anatomy of the liver. Zentralbl. Chir., 125(7):578-86, 2000.

Sexton, C. C. \& Zeman, R. K. Correlation of computed tomography, sonography, and gross anatomy of the liver. A. J. R. Am. J. Roentgenol., 141(4):711-8, 1983.

Soyer, P.; Bluemke, D. A.; Choti, M. A. \& Fishman, E. K. Variations in the intrahepatic portions of the hepatic and portal veins: findings on helical CT scans during arterial portography. A. J. R. Am. J. Roentgenol., 164(1):103-8, 1995.

van Leeuwen, M. S.; Noordzij, J.; Hennipman, A. \& Feldberg, M. A. Planning of liver surgery using three dimensional imaging techniques. Eur. J. Cancer, 31A(7-8):1212-5, 1995.

Wu, T. C.; Lee, R. C.; Chau, G. Y.; Chiang, J. H. \& Chang, C. Y. Reappraisal of right portal segmental ramification based on 3-dimensional volume rendering of computed tomography during arterial portography. J. Comput. Assist. Tomogr., 31(3):475-80, 2007.

\section{Dirección para correspondencia:}

César Augusto Durand López

Cirujano del Hospital de Emergencias "José Casimiro Ulloa"

Profesor de Anatomía Humana, Facultad de Medicina

Universidad Ricardo Palma

Hospital de Emergencias

Av. Roosevelt 6355

Miraflores

Lima- PERÚ

Received: 15-01-2017

Accepted: 24-04-2017

Email: cesaraugustoo1@gmail.com 